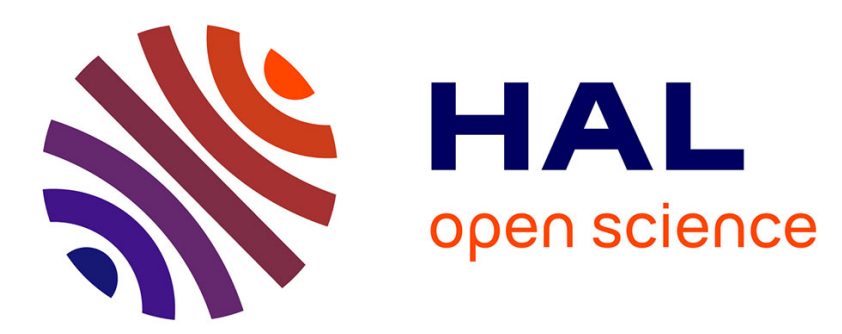

\title{
Integrating Visual and Geometric Consistency for Pose Estimation
}

\author{
Huiqin Chen, Emanuel Aldea, Sylvie Le Hégarat-Mascle
}

\section{To cite this version:}

Huiqin Chen, Emanuel Aldea, Sylvie Le Hégarat-Mascle. Integrating Visual and Geometric Consistency for Pose Estimation. 2019 16th International Conference on Machine Vision Applications (MVA), May 2019, Tokyo, Japan. pp.1-5. hal-02185033

\section{HAL Id: hal-02185033 https://hal.science/hal-02185033}

Submitted on 16 Jul 2019

HAL is a multi-disciplinary open access archive for the deposit and dissemination of scientific research documents, whether they are published or not. The documents may come from teaching and research institutions in France or abroad, or from public or private research centers.
L'archive ouverte pluridisciplinaire HAL, est destinée au dépôt et à la diffusion de documents scientifiques de niveau recherche, publiés ou non, émanant des établissements d'enseignement et de recherche français ou étrangers, des laboratoires publics ou privés. 


\title{
Integrating Visual and Geometric Consistency for Pose Estimation
}

\author{
Huiqin Chen, Emanuel Aldea, Sylvie Le Hégarat-Mascle \\ SATIE - CNRS UMR 8029, Paris-Sud University, Paris-Saclay University \\ Building 660, rue Noetzlin, Gif-sur-Yvette 91190, France \\ \{huiqin.chen, emanuel.aldea, sylvie.le-hegarat\}@u-psud.fr
}

\begin{abstract}
In this work, we tackle the problem of estimating the relative pose between two cameras in urban environments in the presence of additional information provided by low quality localization and orientation sensors. An M-estimator based approach provides an elegant solution for the fusion between inertial and vision data, but it is sensitive to the prior importance of the visual matches between the two views. In addition to using cues extracted from local visual similarity, we propose to rely at the same time on learned associations provided by the global geometrical coherence. A conservative weighting scheme for combining the two types of cues has been proposed and validated successfully on an urban dataset.
\end{abstract}

\section{Introduction}

This paper aims at exploiting and improving the fusion of pose priors from sensors (GPS, accelerometers, gyroscopes, magnetometers) and image features for the estimation of relative camera pose. The estimation of relative camera pose is a fundamental step for many computer vision tasks, such as structure from motion(SFM), SLAM or object tracking. Traditional pose estimation relies on image features: given a pair of images $\left(I_{a}, I_{b}\right)$, the standard procedure may be described as follows:

- Extract SIFT keypoints for $\left(I_{a}, I_{b}\right)$ and assign a built-in descriptor to each keypoint;

- Construct keypoint correspondences from $I_{a}$ to $I_{b}$ by matching them using SIFT descriptor distance minimization with a rejection threshold based on the ratio of the two smallest distances;

- Calculate the relative pose $(R, t)$ by decomposing the fundamental or essential matrix after having computed it using a robust estimation RANSAC on correspondences, or estimate $(R, t)$ directly using an iterative estimation method such as an Mestimator.

However, this traditional strategy only based on image feature is limited by inaccurate matching due to the presence of repetitive features and occlusions, which often occur in urban scenes. Nowadays, sensors available in GPS receivers or IMUs are widely integrated with camera devices in smart phones. These sensors provide a readily available camera pose information, albeit often very imprecise. Despite its uncertainty, the sensor prior can play a crucial role in improving pose estimation based on image feature.
There exist some fundamental approaches for integrating the pose prior with image information. The authors of 1 fuse the pose prior provided by IMU during the matching step in a process which can be considered as filtering for the relative pose estimation. They constrain the search area for correspondences around the epipolar line defined by sensor data in order to guide the matching. As mentioned in 2], this method is very sensitive to sensor noise.

For many works on visual inertial SLAM using temporal sequences, a common fusion method is to constrain separately the image based estimation and pose prior as prediction step and correction step for Kalman filtering 11, 3. Provided that video sequences cover in detail the area of interest, even pure visual SLAM may provide accurate results for registering cameras in urban scenes 10. However, in our work we study the more constrained scenario in which a single pair of images is available, along with a low quality pose prior provided by low cost GPS and inertial sensors. Indeed, in metropolitan areas video-recording (especially using UAVs) is highly regulated, and even if videos from ground-level dynamic cameras are available, they have often low quality and are heavily occluded.

In the last years, minimizing a loss function including both image features and pose prior by non linear optimization has been shown to be more accurate than Kalman filtering in visual-inertial SLAM 4]. To the best of our knowledge, 2 is the first work who extends a similar idea for the relative camera pose estimation for a single pair of views. Instead of using RANSAC, they propose an algorithm called SOREPP which relies on the fusion of putative correspondences and of noisy pose priors from sensors. In order to be robust to outliers, they use the following M-estimator:

$$
\hat{s}=\underset{s}{\arg \min }\left\{c\left(\sum_{k \in \Omega} w(k)(1-g(k, s))\right)+\lambda(s)^{2}\right\},
$$

where $c$ is a weighting parameter, $\Omega$ is the set of putative correspondences and $w(\cdot)$ their weights, $g(k, s)$ is a Gaussian score evaluated for the correspondence $k$ with respect to the relative pose $s$, and the regularization term $\lambda(s)$ is a distance measure between $s$ and the sensor prior.

The performance of SOREPP depends on the sensor precision as well as on the quality of correspondences in the set $\Omega$ and the values of $w(\cdot)$. If the GPS/IMU uncertainty is too high, the regularization term value $\lambda(s)$ decreases and impacts less the optimization. The presence of a significant ratio of outliers in $\Omega$ makes it difficult for the M-estimator to find good solutions, except with very precise pose priors. In order to guide the M-estimator, SOREPP estimates a correspondence 
weight in the form of a rough approximation of the prior probability of being an inlier [2] (see Section 2.1). Although it has the advantage of being simple, the proposed weighting tends to be unreliable in scenes with repetitive patterns such as urban contexts.

Our work aims to further improve the estimation of weights for correspondences, and therefore to improve the performance of pose estimation based on SOREPP. To this aim, we propose that weights be also based on a global geometry consistency estimated by a deep network recently introduced in 5. Then, we explore how to combine this additional criterion with the weighting strategy based on local classical features in SOREPP. Our main contributions consist in:

- applying jointly a neural network with a pose prior for robust relative camera pose estimation;

- developing an effective correspondence weighting strategy by exploiting SIFT features as well as geometric consistency.

\section{Proposed estimation}

In the following, we discuss the weighting strategy based on local visual features from 2, as well as on global geometry consistency as introduced recently in [5]. The last part of this section highlights how these two weighting strategies guided by fundamentally different objectives may be used jointly in order to obtain robust correspondence weights.

\subsection{Weights from classical features}

A set of putative correspondences $\Omega$ is constructed using the classical SIFT nearest neighbor strategy 6 with a standard ratio threshold of 0.75 . SOREPP defines independently the weight $w(k)$ for each correspondence $k$ based on the SIFT ratio test 6 :

$$
\forall k \in \Omega, w(k)=1-\frac{d_{1 N N}(k)}{d_{2 N N}(k)} .
$$

The underlying assumption is that more distinctive the first nearest neighbor, more likely the matching corresponds to an inlier. However, this cue which is based only on the descriptor appearance, is not robust in presence of repetitive features and occlusion. Furthermore, while decreasing the number of outliers, the nonadaptive ratio threshold reduces as well the number of inliers, an outcome which is undesirable when inliers are scarce. Next we will discuss the weighting strategy based on global geometry consistency which avoids relying on a fixed ratio threshold.

\subsection{Weights from the geometry network}

Download

Source

PDF

Actions

Copy Project Word Count

Sync

Dropbox

Git

GitHub
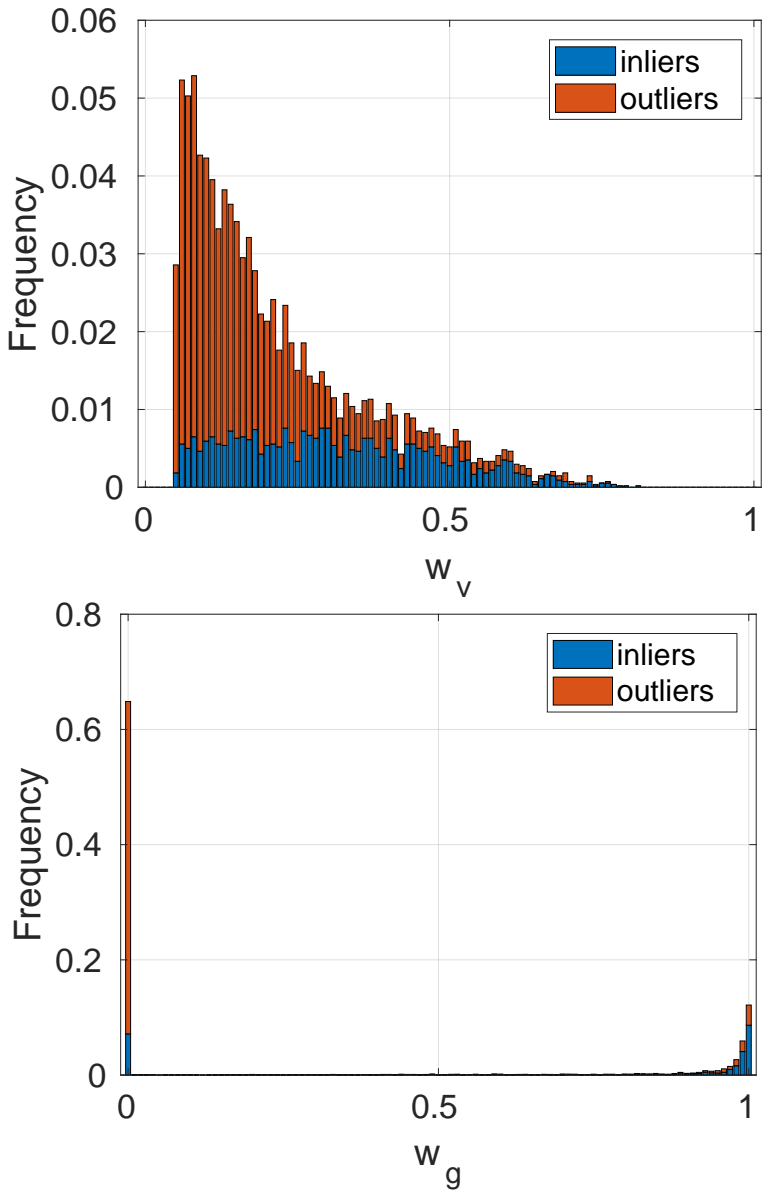

Figure 1: Histogram of inliers and outliers for $w_{v}$ (above) and $w_{g}$ (below). The neural network provides a much crisper output compared to a traditional appearance based evaluation.

Settings Compiler Main document Spell check Autocomplete Auto-close Brackets Code check Editor theme Overall theme Keybindings Font Size Font Family Line Height PDF Viewer Hotkeys

Show Hotkeys

PoseEstimationForSurveillance

Editor mode. 4

Given all coordinates of putative correspondences in $\Omega=\left\{k_{1}, k_{2}, \ldots, k_{i}, \ldots, k_{N}\right\}$, with $k_{i}=$ $\left(x_{a i}, y_{a i}, x_{b i}, y_{b i}\right) \in \Omega$, for an image pair $\left(I_{a}, I_{b}\right)$, which is constructed by classical SIFT nearest neighbor strategy without ratio threshold, a weakly supervised deep network is trained in 5 to estimate a global geometrically consistent weight $w_{k} \in[0,1]$ for each correspondence:

$$
\left\{w\left(k_{1}\right), w\left(k_{2}\right), \ldots, w\left(k_{i}\right), \ldots, w\left(k_{N}\right)\right\}=f_{\Phi}(\Omega),
$$

where $f_{\Phi}(\cdot)$ is the regression of a deep network with parameters $\Phi$. If $w\left(k_{i}\right)=0$, the correspondence $k_{i}$ is likely to be an outlier and classified as such, following the precautionary principle.

The core idea is that inliers are geometrically structured, conversely to outliers. Unlike the weight based on ratio test in Section 2.1 that depends only on the considered correspondence, when considering geometric consistency all correspondences contribute to the 
estimation of $w\left(k_{i}\right)$. Because this globally geometric weight is independent of appearance, it can potentially avoid the appearance ambiguity for repetitive features.

\subsection{Exploiting both cues}

It is useful to exploit jointly the local appearance cue and global geometric consistency cue because of their complementary information and their relative balance in performance and precision. In the following part, different combinations of these cues are investigated and compared. For the sake of simplicity, $w_{v}$ denotes the weight from classical visual features given by Eq. (2), $w_{g}$ the weight from the geometry network given by Eq. (3) and $w^{(i)}$ the combined weight to be investigated for a specific correspondence $k$.

Linear operation. The simplest fusion approach is to consider the average of $w_{g}$ and $w_{v}$ :

$$
w^{(1)}=\frac{w_{g}+w_{v}}{2},
$$

Averaging can be seen as a weight filtering which allows to keep considering correspondences having possible a very low weight $w_{v}$ or $w_{g}$, while reinforcing correspondences with both high weights. In our case, this averaging approach is taken as the baseline.

Regression. Instead of applying a simple averaging operation, it is possible to learn automatically the fusion weight by logistic regression :

$$
w^{(2)}=g\left(w_{g}, w_{v}\right)
$$

where $g(\cdot)$ represents the bivariate logistic regression function.

In order to train the regression model, we choose image pairs in the dataset of [5] and match corners using the classical nearest neighbor SIFT strategy. The values $w_{v}$ are computed during the matching step, whereas values $w_{g}$ are obtained by taking the matched correspondence coordinates for each image pair as the input of neural network model in [5] with the trained model. By choosing a fixed Sampson distance threshold of matching points to the epipolar line, each correspondence is labeled as 1 for inliers or 0 for outliers. As expected, in our setting the resulting weight is close to 1 when $w_{v}$ is larger than 0.8 irrespective of the value of $w_{g}$. This seems to be reasonable since for few outliers the ratio test is higher than 0.8 according to Fig. 11 upper histogram. Compared to averaging, regression tends overall to increase the combined weight, which might increase more the influence of potential inliers but also that of some outliers.

Conservative weighting. The previous strategies introduce more or less some confusion between inliers and outliers in terms of weighting for the M-estimator based optimization. In order to be stricter, we propose to adopt a conservative weighting based on a pessimistic function such as $\min \left(w_{g}, w_{v}\right)$. One significant consequence is that, in contrast to previous strategies, the min operation discards entirely the correspondences with $w_{g}=0$, which exhibit a high ratio of outliers.

Since $w_{v}$ seems to be more unreliable according to Fig. 11, the symmetric weight from visual cues denoted by $w_{v s}$ (computed by interchanging for the association the source and destination images in the pair) is also taken into account in order to constrain more the influence of visual part, and $w_{v}$ is substituted by $\min \left\{w_{v}, w_{v s}\right\}$. However, the geometry network exhibits already a conservative behaviour in the way it outputs the weights (see Fig. 1, lower histogram), thus the symmetric weight from the network denoted by $w_{g s}$ is used conversely in order to allow more prospective points identified by geometric coherence: $\max \left\{w_{g}, w_{g s}\right\}$. Finally, by taking into account the particular behaviour of the two weighting algorithms, the fusion weight is computed by the following formula:

$$
w^{(3)}=\min \left\{\max \left\{w_{g}, w_{g s}\right\}, \min \left\{w_{v}, w_{v s}\right\}\right\},
$$

The result in Eq. (6) is also due to the fact that the loss function used in the neural network encourages a crisp decision regarding the nature of each input observation, a behavior which is clearly visible as well in Fig. 1] when comparing the geometry weighting with the histogram of $w_{v}$ values.

\section{Experiments}

In the experimental part, we evaluate the performance of the weighting strategies introduced in the previous section, and we compare them to SOREPP algorithm [2] and to the geometric network [5] on a dataset collected in a challenging urban environment. We start by presenting the dataset, then we introduce the implementation details followed by the evaluation metric. The quantitative details are then discussed.

\subsection{Dataset}

We collected an urban scene dataset containing 32 images acquired with a smart phone in front of a major railway station, some of the images being taken at ground level and some from the upper floors of a building. As in 2, the GeoCam application was used to record in each image header the approximate pose provided by the embedded sensors. The ground truth was constructed by feeding all the images into VisualSFM [7]. The relative poses were varied gradually in order to ensure a high-quality VisualSFM estimation, and also to provide image pairs with varying degrees of difficulty for our pose estimation problem. The dataset is provided to the academic community 1

\subsection{Implementation}

Given an image pair from the dataset, we compute two correspondence sets $\Omega_{1}$ and $\Omega_{2}$ using the SIFT nearest neighbor strategy with different ratio test thresholds. A standard ratio test threshold 0.75 is chosen for $\Omega_{1}$. For $\Omega_{2}$, we choose a very high value 0.95 which almost amounts to cancelling the ratio test, but it actually still helps eliminating a fair number of correspondences. We compute the weight $w_{v}$ as in Eq. (3) for each correspondence in $\Omega_{1}$ and $\Omega_{2}$ during the matching step. Furthermore, we take all normalized matching point coordinates in $\Omega_{2}$ as the input of the geometric network in [5] and we get a geometric weight

${ }^{1}$ The data used in this work may be found at: hebergement.u-psud.fr/emi/S2UCRE/ChenMVA19.zip http:// 
$w_{g}$ for each correspondence in $\Omega_{2}$. We use $\Omega_{2}^{*}$ to denote the subset of $\Omega_{2}$ where $w_{g}$ is greater than 0 and each correspondence in $\Omega_{2}$ is normalized. We applied the model trained by the authors of [5] on outdoor scenes. At the same time, we also compute three versions of fusion weight $w^{(1)}, w^{(2)}$ and $w^{(3)}$ for $\Omega_{2}$ separately with Eq. (4), (5) and (6).

According to the various inputs and algorithms, we classify the different estimation methods as shown in Tab 1 .

Table 1: Estimation method

\begin{tabular}{c|cc}
\hline \hline Name & Algorithm & Input \\
\hline Sensor & - & - \\
SIFT-RANSAC & RANSAC & $\Omega_{1}$ \\
GEO-RANSAC [5] & RANSAC & $\Omega_{2}^{*}$ \\
SIFT-SOREPP [2] & SOREPP & $\Omega_{1}, w_{v}$, sensor \\
GEO-SOREPP & SOREPP & $\Omega_{2}, w_{g}$, sensor \\
Ours-LO & SOREPP & $\Omega_{2}, w^{(1)}$, sensor \\
Ours-REG & SOREPP & $\Omega_{2}, w^{(2)}$, sensor \\
Ours-CNSV & SOREPP & $\Omega_{2}, w^{(3)}$, sensor \\
\hline \hline
\end{tabular}

\subsection{Evaluation metric}

Given the estimated rotation matrix and translation vector $\left(R_{e s}, t_{e s}\right)$ and the ground truth $\left(R_{g d}, t_{g d}\right)$ for relative camera poses, we compute the rotation error $\delta R$ and translation error $\delta t$ separately for the evaluation. For $\delta R$, we firstly compute the relative rotation matrix $\Delta R$ between $R_{e s}$ and $R_{g d}$, with $\Delta R=R_{e s}^{T} \cdot R_{g d} . \Delta R$ can be represented by a rotation of angle $\phi$ around a vector $v$. Smaller is $\phi$, closer $R_{e s}$ is to $R_{g d}$. Thus the extent of the rotation error $\delta R$ can be approximated by $\phi$ which is computed as (see page 584 in [8]):

$$
\delta R \approx \phi=\arccos \left(\frac{\operatorname{Tr}(\Delta R)-1}{2}\right),
$$

with $\operatorname{Tr}(\cdot)$ the trace of a matrix.

The translation error $\delta t$ is computed as the angle between $t_{e s}$ and $t_{g d}$ :

$$
\delta t=\arccos \left(\frac{t_{e s} \cdot t_{g d}}{\left\|t_{e s}\right\|\left\|t_{g d}\right\|}\right),
$$

In the following, we take the pose estimation error as the maximum value between $\delta R$ and $\delta t$. To evaluate the performance of each method, we compute the cumulative curve of pose estimation error taken, as previously performed in [5] and in 9]. For each method, the corresponding curve presents the percentage of image pairs whose pose is successfully estimated with respect to a given error threshold. As a qualitative result, we illustrate in Fig. 2 a relative pose estimation for an image pair, and the corresponding epipolar lines and epipole locations for the different methods considered.

\subsection{Quantitative evaluation}

The evaluation results are presented in Fig. 3. When comparing the weighting strategies and the other methods, the Ours-CNSV approach which uses the conservative fusion rule shows the best performance. The important role of the noisy pose priors provided by sensors is confirmed by the SOREPP based estimation (SIFT-SOREPP) which improves over the pure vision based algorithms, namely classical SIFT-RANSAC and the geometry based network (GEO-RANSAC). The linear weighting and the bivariate regression weights are less effective in supporting the M-estimator, especially in the high precision range (error smaller than 5 degrees) which is desirable for the accurate localization of elements of interest in the camera fields of view. Our experiments confirm systematically that relying on the more permissive $\Omega_{2}$ threshold for visual filtering is more effective than using the stricter $\Omega_{1}$, because the global geometric consistency provided by the neural network identifies more reliably the inliers than the local fixed ratio test threshold used classically for SIFT matching.

\section{Conclusion}

For the relative pose estimation problem, we proposed a strategy to rely at the same time on local information provided by visual similarity, and by a global geometrical coherence of the transformation between two views. We combined the two types of cues along with localization and orientation information within an M-estimator, with excellent results on real data acquired in an urban scenario.

In future work, we intend to formalize the usage of prior knowledge in the combination process in order to extend it for other sources of information assessing the reliability of interest point matches, i.e. belonging to the same semantic category or exhibiting local similarity in terms of a learned function.

\section{Acknowledgements}

This work was funded by the French National Research Agency grant ANR-16-SEBM-0001 (the "S ${ }^{2}$ UCRE" project) within the French-German funding framework "Safety and Security of Urban Crowded Environments".

\section{References}

[1] DR Wong, MP Hayes and al.: "IMU-aided SURF feature matching for relative pose estimation," Image and Vision Computing New Zealand (IVCNZ), 2010 25th International Conference of, pp.1-6,IEEE, 2010.

[2] Y Goldman, E Rivlin,I Shimshoni: "Robust epipolar geometry estimation using noisy pose priors," Image and Vision Computing, vol.67, pp.16-28, 2017.

[3] R Brockers, S Susca, D Zhu and al.: "Fully self-contained vision-aided navigation and landing of a micro air vehicle independent from external sensor inputs," $U n$ manned Systems Technology XIV, vol.8387, pp.83870Q, International Society for Optics and Photonics, 2012.

[4] S Leutenegger, S Lynen, M Bosse and al.: "Keyframebased visual-inertial odometry using nonlinear optimization," The International Journal of Robotics Research, vol.34, n.3, pp.314-334, SAGE Publications Sage UK: London, England, 2015. 


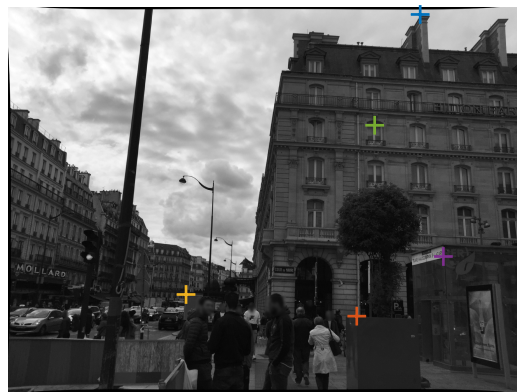

(a) Image at ground level

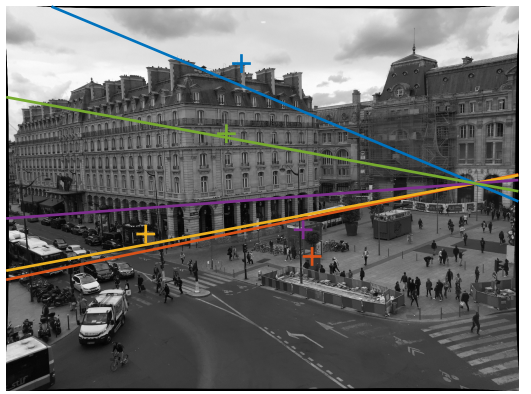

(d) GEO-RANSAC 5

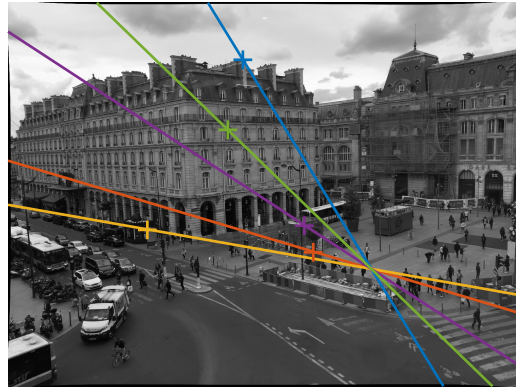

(b) Ground truth

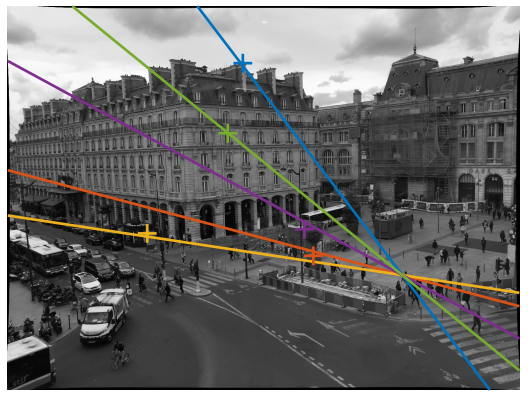

(e) SIFT-SOREPP 2

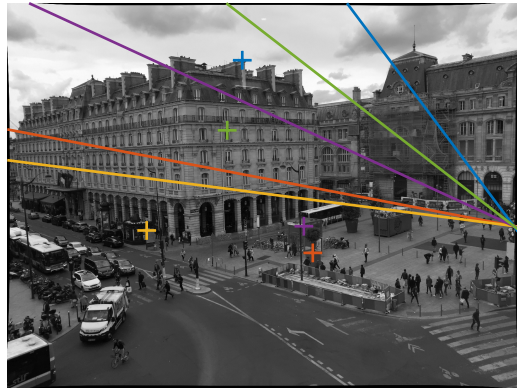

(c) Sensor provided pose

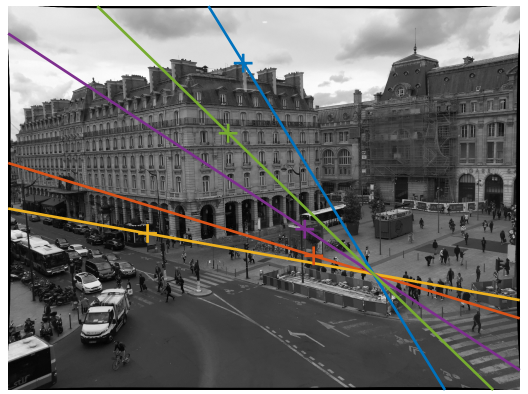

(f) Ours-CNSV

Figure 2: Illustration of pose estimation results between a ground level view (Fig. 2a) and an overview camera. The ground truth (Fig. 2b) is computed using SfM on a larger set of images. The quality may be assessed visually based on the position of the manually defined control points with respect to their epilines, and based on the location of the epipole.

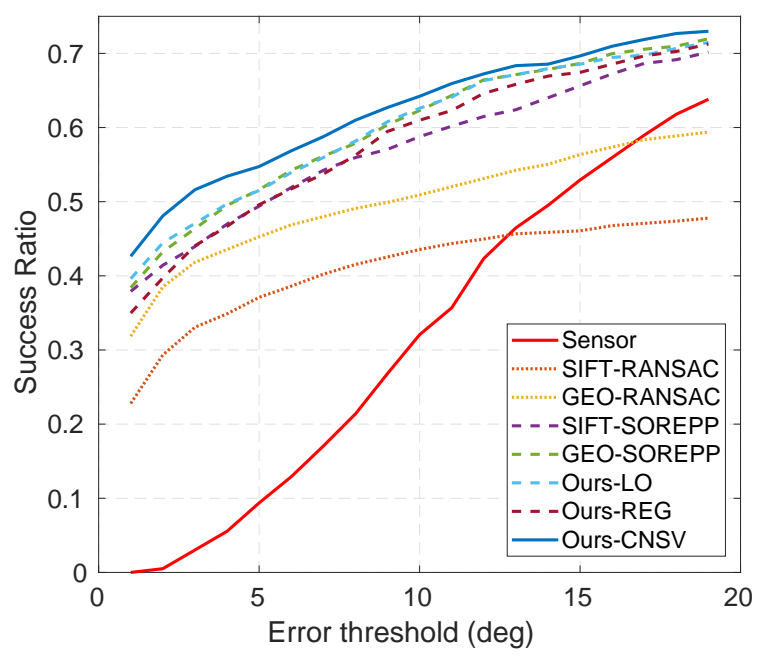

Figure 3: Rotation and translation success ratio of various pose estimation algorithms depending on a given error threshold.

[5] KM Yi, E Trulls, and Y Ono , V Lepetit and al.: "Learning to Find Good Correspondences," Proceed- ings of the 2018 IEEE/CVF Conference on Computer Vision and Pattern Recognition (CVPR), CONF, 2018.

[6] DG Lowe: "Distinctive image features from scale invariant keypoints," International journal of computer vision, vol.60, n.2, pp.91-110, 2004.

[7] C Wu: "VisualSFM: A visual structure from motion system," 2011.

[8] R Hartley, A Zisserman: "Multiple view geometry in computer vision," Cambridge university press,2003.

[9] JW Bian, WY Lin, Y Matsushita and al.: "Gms: Grid-based motion statistics for fast, ultra-robust feature correspondence," Computer Vision and Pattern Recognition, 2017 IEEE Conference on, pp.2828-2837, IEEE, 2017.

[10] T Pollok, E Monari: "A visual SLAM-based approach for calibration of distributed camera networks," 13th IEEE International Conference on Advanced Video and Signal Based Surveillance, AVSS, pp.429-437, IEEE, 2016.

[11] M Pollefeys, D Nistér, J-M Frahm, A Akbarzadeh, P Mordohai, B Clipp, C Engels,D Gallup, S-J Kim, P Merrell and al.: "Detailed real-time urban 3d reconstruction from video," International Journal of Computer Vision, vol.78, n.2-3, pp.143-167, Springer, 2008. 